\title{
Jean Hamburger, la médecine et la science
}

Jean-François Bach

Personne mieux que Jean Hamburger ne peut symboliser la communauté de pensée et d'action de la médecine et de la science dont cette revue s'est fait le brillant porte-parole. Ce fut, en effet, tout au long de sa vie, l'idée directrice permanente de Jean Hamburger que d'introduire la rigueur du raisonnement scientifique en médecine et de chercher dans la recherche fondamentale les armes du progrès médical. La science ne se réduit pas à la réalisation d'expériences complexes ni à l'utilisation d'appareils sophistiqués. Comme la médecine, elle repose en premier lieu sur les deux grandes qualités de l'esprit que sont la rigueur et l'imagination. La médecine sans

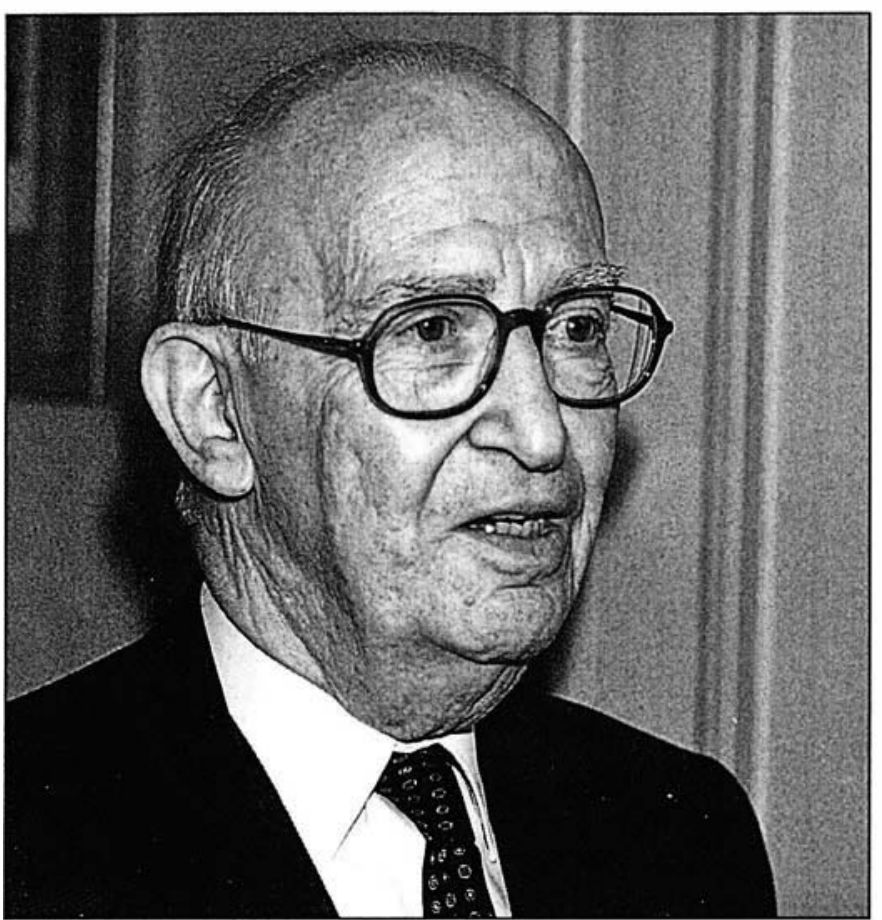
science est vouée à l'immobilisme, avec le risque incessant de voir resurgir un certain obscurantisme. La science biologique sans ouverture médicale perd une de ses plus grandes dimensions. C'est dans cet esprit que Jean Hamburger eut l'idée avec Henri Flammarion de créer ce qui allait devenir médecine/sciences. C'est autour de ce même idéal qu'il a construit toute sa vie de médecin et de chercheur puis de philosophe. Démarche admirable si l'on pense qu'à l'époque où elle fut entreprise, au début des années 1950, la médecine française était exsangue et la recherche médicale quasiment inexistante.

L'œuvre de Jean Hamburger s'inscrit dans ce contexte. Parti de la physiologie et de l'analyse anatomo-clinique, il franchit à pas de géant tout le champ de la néphrologie, dont il est l'incontestable père iondateur, jusqu'à ses confins immunologiques et génétiques. On ne compte pas les découvertes originales, les grandes premières, les nouvelles idées qui ont émaillé ces 40 années passées à l'hôpital Necker. Il convient d'en détacher au plus haut niveau l'élan décisif donné aux transplantations rénales. Jean Hamburger fut un des tout premiers, dans l'incrédulité et l'indifférence générales, à en percevoir les promesses, à tenter avec succès les greffes les plus audacieuses, à réfléchir aux problèmes immunologiques considérables posés par le rejet et à poser les questions éthiques essentielles soulevées par le choix du donneur. Son nom restera inscrit en lettres d'or dans l'histoire des transplantations.

L'apport au développement de la réanimation médicale et de la néphrologie est également d'importance majeure. Il joua un rôle prépondérant dans la dissection des anomalies métaboliques conduisant aux états de deshydratation et d'hyperhydratation cellulaires. Il proposa une nouvelle classification des maladies rénales en décrivant plusieurs maladies majeures jusque-là non reconnues. Il promut avec détermination le développement du rein artificiel avec tous les problèmes scientifiques, économiques et éthiques qui étaient alors rencontrés, il lança des recherches novatrices sur les maladies auto-immunes, notamment le diabète.

Au-delà de ces contributions, Jean Hamburger demeurera un des grands maîtres à penser de la médecine de notre temps. Il fut un des premiers à comprendre la révolution irréversible de la pratique clinique, transformée par un biologie triomphante sans pour autant négliger la dimension humaine. Le service de néphrologie qu'il dirigea pendant près de 40 ans était un modèle de qualité clinique où s'associaient au plus haut niveau une compétence médicale imaginative, un support technologique hors pair et une relation privilégiée avec le malade.

Jean Hamburger n'était pas seulement un chercheur créatif et déterminé, un médecin sensible et courageux, un mâ̂tre stimulant et chaleureux. Il était aussi présent sur tous les fronts où pouvait être défendu le développement de la recherche médicale. Il avait contribué à créer la Fondation pour la Recherche Médicale et l'INSERM. Il défendait toujours avec ardeur et talent le renom de la recherche médicale française à l'étranger. Jean Hamburger était un homme complet, sachant atteindre une quasi-perfection dans chacune de ses activités. Un homme unique dont la disparition nous plonge dans une grande tristesse, proche du désarroi. La médecine française doit se montrer digne de l'exemple exceptionnel laissé par Jean Hamburger.

\section{J.F. Bach}

Inserm U. 25, Immunologie clinique, hôpital Necker, 161, rue de Sèvres, 75743 Paris Cedex 15, France. 